\title{
Degradation of Polystyrene by Tropical Bacterial and Fungal Isolates
}

\section{${ }^{1}$ AKERELE, OS; ${ }^{1 *}$ BURAIMOH, OM; ${ }^{2}$ HUMPHREY, I; ${ }^{1}$ ILORI, MO}

\author{
${ }^{1}$ Department of Microbiology, Faculty of Science, University of Lagos, Akoka, Lagos, Nigeria \\ ${ }^{2}$ Department of Physics, Faculty of Science, University of Lagos, Akoka, Lagos, Nigeria \\ *Corresponding Author Email: oburaimoh@unilag.edu.ng; marianiks@yahoo.com; Tel: +2348033849915 \\ Other Authors Email: odun.akerelesam@gmail.com; ihumphrey@unilag.edu.ng; olusojiilori@yahoo.com
}

\begin{abstract}
Plastic waste is one of the major contributing factors to the growth of municipal solid wastes globally. It is a threat to public health and negatively impact the aquatic and terrestrial animals due to its persistence in the environment. Bacterial and fungal isolates were tested for their ability to degrade polystyrene. The cultures were grown on mineral salts medium supplemented with polystyrene as the sole carbon source in $250 \mathrm{~mL}$ conical flasks. The polystyrene reduced from $1 \mathrm{~g}$ (week 0 ) to $0.7 \mathrm{~g}$ (week 7). Statistical analysis of variance (one-way ANOVA) shows a significant difference between the reduction in weight of polystyrene in the experimental flask compared to the control $(\mathrm{P}<0.05)$. Gas chromatography mass spectrometry analysis of biodegradation products shows that some toxic polystyrene constituents such as O-xylene, cis-9-hexadecenal and 3-phenol pentadecyl were removed.
\end{abstract}

\section{DOI:https://dx.doi.org/10.4314/jasem.v25i9.26}

Copyright: Copyright (C) 2021 Akerele et al. This is an open access article distributed under the Creative Commons Attribution License (CCL), which permits unrestricted use, distribution, and reproduction in any medium, provided the original work is properly cited.

Dates: Received: 09 May 2021; Revised: 12 August 2021; Accepted: 12 September 2021

Keywords: Digestate, Polystyrene, municipal, O-xylene, degrade

Plastic is a key factor in the waste generated on a global level. This is due to the wide variety of applications by food, cosmetic, textile and other industries. (Urbanek, 2018). Large amounts of plastics are used in packing films, wrapping materials, shopping and garbage bags, fluid containers, toys, household industrial products, and building materials. More than 300 million metric tons of plastic are produced globally each year (Bratovcic, 2019), with nearly half of this volume being used once and discarded within a year, and Up to $10 \%$ of plastic waste is managed through recycling (Garcia and Robertson, 2017; Lettieri and Al-salem, 2011). However, the harmful impacts of plastic usage outweigh the benefits (Bratovcic, 2019). Plastic waste is a key environmental threat due to its nonbiodegradability, posing a health risk to both aquatic and terrestrial animals (Chae and An, 2017). The extensive use of plastics has led to the increased, and continuous exposure of humans to components such as additives and plasticizers which is of great health concern. When accidentally ingested, micro plastics may be caught in the gastrointestinal system (Compa, 2018). The sperm whale which was found on the shore of Eastern Indonesia was reported to have consumed 6 $\mathrm{kg}$ of plastics, which included 25 plastic bags, 115 cups, and plastic bottles (www.natgeo.org/plastics). Biomonitoring studies have shown the existence of a steady-state plastic component concentration in the human body, thereby reflecting the continuing equilibrium of continuous exposure, metabolism, and excretion of these compounds (van Cauwenberghe and Janssen, 2014). Several hazardous and ecologically damaging effects are the result of plastic pollution in the aquatic environment. Close to three hundred (300) species of aquatic animals have been reported to ingest or get ensnared in plastic debris, which leads to marred movement and feeding, lacerations, ulcers, decreased reproductive output, and death (Romeo et al., 2015). The degree of plastic waste in marine habitats have been measured, with most of it concentrated on litter accumulation on beaches. (Jonas et al., 2019). This is possibly because plastics generally float and, thus, appear to build on the beaches. Nearly three quarters of all the debris washed up onshore is made of plastic (Foley et al., 2018). Polystyrene (PS) is one of the common petroleum-based synthetic polymers made from styrene (vinyl benzene) monomer. It is commonly used in the manufacturing of disposable food packaging boxes, laboratory ware, disposable cups, and packaging materials and in certain electronic uses. This is due to its properties such as light weight, stiffness, and excellent thermal insulation (Jia, 2016). Two classes of polystyrene are extruded polystyrene (XPS) and expanded polystyrene (EPS). The main difference between the two lies in their density. The 
extruded type is denser than expanded polystyrene (www.chemicalsafetyfacts.org). The fate of plastic material is municipal solid waste (Horodytska, 2018). Some of the methods such as incineration or mechanical recycling has been proposed for the management of plastic waste. Meanwhile, thermal and chemical breakdown can lead to harmful and toxic emissions, and products like styrene, acrolein, benzene and toluene (Horodytska, 2018). In addition, these two processes are costly and may not be economically viable (Ragaert et al., 2017). Although biodegradation of styrene by microbial action exists, however, reports on the microbial biodegradation of polystyrene are scarce (Aruna and Shanthi, 2015). Therefore, the present study examined the ability of bacterial and fungal isolates from digestate, cow dung, and abattoir effluent to degrade polystyrene.

\section{MATERIAL AND METHODS}

Preparation of sample: New plastic (polystyrene) was obtained from Bariga market, Lagos, Nigeria and subsequently identified using resin identification code (http://guides.stopwaste.org). The polystyrene was ground with a mill, sieved with a wire mesh $(0.3 \mathrm{um})$, stored in a clean sterilizable container, and sterilized at $121^{\circ} \mathrm{C}$ for 10 minutes. Thereafter, it was used for in vitro laboratory degradation experiment.

Isolation of Microorganisms: Isolation from Cow Dung: Fresh cow dung was obtained from a cattle farm at Gbagada, Lagos state Nigeria and transported in an ice pack $\left(4^{\circ} \mathrm{C}\right)$ to the department of microbiology, University of Lagos for immediate analysis. One gram was measured into a test tube containing sterile distilled water $(9 \mathrm{~mL})$. It was dislodged appropriately by shaking to homogenize the suspension $\left(10^{-1}\right)$. Thereafter, $1 \mathrm{~mL}$ of aliquot was taken from the first dilution (i.e., $10^{-1}$ ) and transferred into another tube containing $9 \mathrm{~mL}$ of sterile distilled water to achieve a dilution of $10^{-2}$. Further dilutions were made until $10^{-5}$ dilution. These dilutions (i.e., $10^{-2}, 10^{-3}, 10^{-4}$ and $10^{-5}$ ) were plated on nutrient agar. For inoculation, $0.1 \mathrm{~mL}$ were taken from each of the selected dilutions and transferred to each of the labelled corresponding plates respectively. Plates of bacteria, were incubated at 37 ${ }^{\circ} \mathrm{C}$ for $24 \mathrm{~h}$. Distinct colonies were picked from the primary plates and streaked out on freshly prepared plates to obtain pure colonies. Then the pure isolates were stored in agar slants at $4{ }^{\circ} \mathrm{C}$.

Isolation from Digestate: Bacterial species were isolated from digestate (materials remaining after anaerobic digestion). The improvised anaerobic digester was carefully opened at an angle of $30^{\circ}$, thereafter, appropriate quantity of the digestate was collected with a sterile spatula into a sterile sample bottle. The digestate sample was processed immediately after collection. One gram was measured into a test tube containing sterile distilled water $(9 \mathrm{~mL})$ and serial dilutions were carried out as explained above. Plates were incubated at $35{ }^{\circ} \mathrm{C}$ for $24 \mathrm{~h}$. The plates were observed for growth after $24 \mathrm{~h}$ of incubation in anaerobic jar. Pure cultures were obtained by sub-culturing on nutrient agar, they were subsequently characterized by their morphological characteristics and biochemical reactions.

Biochemical Characterization: After an appropriate incubation period, the shape, size, pigmentation, elevation, and marginal characteristics of the bacterial species were examined on the nutrient agar plates. Pure cultures of bacterial isolates were identified based on their morphology and biochemical characterization. The following tests were performed on each isolate; spore forming test, motility test, catalase, oxidase, citrate utilization test, Nitrate reduction test, Methyl-Red Voges Proskauer Test, casein hydrolysis, gelatin hydrolysis, starch hydrolysis, Urease activity, carbohydrate utilization test, Growth on MacConkey Agar, indole test and hydrogen sulphide production.

Preparation of Aspergillus niger Strain R62 (MK542010): Aspergillus niger strain R62 MK542010 was isolated from abattoir effluent at Kara market Ogun State Nigeria. It was cultivated using Sabouraud dextrose agar (SDA) and potato dextrose agar (PDA). Morphological features of colonies as well as microscopic characteristics were studied. Further identification was by amplification and sequencing of the ITS gene of the fungus. The ITS gene sequences obtained from the isolate were deposited in the NCBI database and are available under accession number MK542010

Degradation studies: Mineral salts medium $(100 \mathrm{~mL}$, $\mathrm{pH}$ 7.2) was prepared into $250 \mathrm{~mL}$ conical flasks. One gram of polystyrene was weighed into each of the conical flasks as the sole source of carbon. Sterilization was done at $121^{\circ} \mathrm{C}$ for $15 \mathrm{~min}$ and was allowed to cool to room temperature. Inoculation was done by introducing $1.0 \mathrm{~mL}$ of selected isolates. There were two controls; the first contain mineral salts medium (MSM) only, while the second contained mineral salts medium (MSM) and substrate (Polystyrene). The flasks were covered with sterile cotton plug and incubation was done in a shaker (140 $\mathrm{rpm})$ at $37^{\circ} \mathrm{C}$ for seven weeks. Harvesting was done weekly. The metabolic products were determined using gas chromatography mass spectrometry Shimadzu Training Centre for Analytical Instruments 
(STC), Lagos Nigeria. For degradation studies using Aspergillus strain, the medium containing mineral salts medium $(150 \mathrm{~mL}, \mathrm{pH}$ 6.06) was prepared into $250 \mathrm{~mL}$ conical flasks with sterilized dried cow dung as a nitrogen source. One and a half gram $(1.5 \mathrm{~g})$ of polystyrene was weighed into each of the conical flasks as the sole source of carbon. The flasks were sterilized at $121{ }^{\circ} \mathrm{C}$ for 15 minutes and allowed to cool to room temperature. Inoculation was done by introducing $1.0 \mathrm{~mL}$ of the washed fungus spore into each of the conical flasks except the control. The flasks were plugged with sterile cotton wool and kept in incubator with shaker (ZHP-100, PEC medical CHINA) set to rotate at $130 \mathrm{rpm}$ at $28{ }^{\circ} \mathrm{C}$ for eight weeks.

Measurement of Reduction of Polystyrene Residues: The culture medium containing the mineral salts medium, and the substrate was filtered with preweighed filter paper (Fisher brand, grade P8, 20-25 um particle retention). After the filtration, it was washed, and oven dried at $100{ }^{\circ} \mathrm{C}$ for 15 minutes. Then, the weight was measured with electronic weighing balance $\left(\right.$ Ohaus $\left.{ }^{\circledR}\right)$. The weight was recorded accordingly. The weight of the substrate (polystyrene) remaining in the medium was obtained by subtracting the weight of the filter paper from the weight of the dried substrate plus filter paper. This was repeated for each sample after harvesting every week throughout the period of the experiment (Buraimoh et al., 2015).

Total Viable Count: The growth of the bacterial isolates in the polystyrene medium was monitored by observing the Total viable count (TVC) at seven days' interval. One milliliter $(1 \mathrm{~mL})$ each of the bacterial suspension was initially introduced into each of the medium. One $\mathrm{ml}$ was aseptically pipette into a tube containing $9 \mathrm{~mL}$ sterile distilled water making $10^{-1}$ dilution. It was dislodged appropriately by shaking to homogenize the suspension $\left(10^{-1}\right)$. Thereafter, $1 \mathrm{~mL}$ of aliquot was taken from the first dilution (i.e., $10^{-1}$ ) and transferred into another tube containing $9 \mathrm{~mL}$ sterile distilled water to obtain a dilution of $10^{-2}$. Further dilutions were made until $10^{-6}$ dilution. For TVC, 0.1 $\mathrm{mL}$ was taken from $10^{-4}$ dilution and was inoculated on to a nutrient agar plate. Incubation was done at 37 ${ }^{\circ} \mathrm{C}$ for $24 \mathrm{~h}$.

Gas Chromatography Mass Spectrometry: Gas Chromatography Mass Spectrometry (GC-MS) analysis were performed in a gas chromatograph model GC17 (Shimadzu, Japan) at Shimadzu Training Centre for Analytical Instruments (STC) Lagos. The flow rate of the carrier gas, helium (He) was $1 \mathrm{~mL}$ $\min ^{-1}$, split ratio was $1: 1$. The injector temperature was adjusted to $250{ }^{\circ} \mathrm{C}$, while the detector temperature was set to $280^{\circ} \mathrm{C}$. The column temperature was maintained at $60{ }^{\circ} \mathrm{C}$ for $2 \mathrm{~min}$ followed by linear programming to raise the temperature from $60^{\circ} \mathrm{C}$ to $120^{\circ} \mathrm{C}$ (at $4{ }^{\circ} \mathrm{C}$ min ${ }^{-1}$ with 3 min hold time), $120^{\circ} \mathrm{C}$ to $170{ }^{\circ} \mathrm{C}$ (at $6^{\circ} \mathrm{C}$ $\mathrm{min}^{-1}$ with $1 \mathrm{~min}$ hold time) and $170{ }^{\circ} \mathrm{C}$ to $290{ }^{\circ} \mathrm{C}$ (at $10^{\circ} \mathrm{C}$ min $^{-1}$ with 1 min hold time). The transfer line was heated at $280{ }^{\circ} \mathrm{C}$. Two microliter of sample was used for analysis. Mass spectra were obtained in scan mode $(70 \mathrm{eV})$; in the range of $45-700 \mathrm{~m} / \mathrm{z}$

\section{RESULTS AND DISCUSSION}

Five bacterial isolates and a fungus (Aspergillus niger strain R62 MK542010) obtained from digestate, cow dung and abattoir effluent, were used for this study. The morphological examination shows that isolates OS1, OS2 and 2OS were gram positive rods and able to form endospores. Isolates CDOS 2 and CDOS 3 were gram negative rods. The five isolates were presumptively identified based on their morphological and biochemical characteristics as Bacillus sp., Paenibacillus sp., Bacillus sp., Pseudomonas sp. and Alcaligenes.

Reduction in Weight of Polystyrene: The results of the dry weight method carried out to determine the reduction of polystyrene in the growth medium showed that in the flask inoculated with isolates OS1, the polystyrene reduced from $1 \mathrm{~g}$ to $0.7 \mathrm{~g}$ while the flask with CDOS2 reduce to $0.72 \mathrm{~g}$. Figure 1 showed the summary of residual polystyrene in the experimental flasks

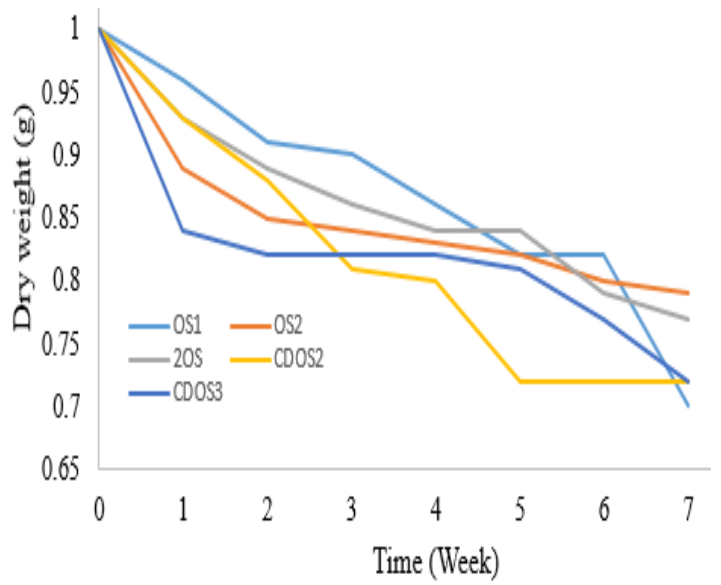

Fig 1: Reduction in weight of polystyrene residue: Legend: OS1Bacillus sp., OS2- Paenibacillus sp., 2OS - Bacillus sp., CDOS2 Pseudomonas sp., CDOS3 - Alcaligenes sp.

Total Viable Count: The results of the total viable count determined showed that isolate OS2 and 2OS was above 300 colony counts in week 5 . By the end of week 7 the least colony count was 102 (isolate CDO2) as shown in figure 2 below 


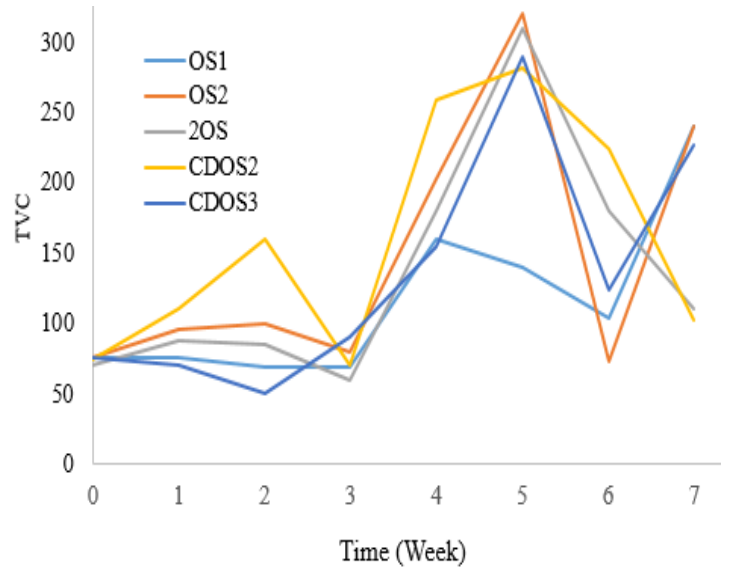

Fig 2: Total viable count of bacteria isolates on polystyrene medium. Legend: OS1: Bacillus sp., OS2: Paenibacillus sp., 2OS: Bacillus sp., CDOS2: Pseudomonas sp., CDOS3: Alcaligenes sp.

pH Changes in the Medium Containing Polystyrene: The $\mathrm{pH}$ of the culture media was taken at two days' interval. Slight changes in $\mathrm{pH}$ from 7.2 to $7.56,7.2$ to $7.43,7.21$ to 7.41 , and 6.24 to 7.04 was observed in the culture media containing isolates 2OS, CDOS2, CDOS3 and A. niger strain R62 MK542010 respectively.

Gas Chromatography - Mass Spectrum Analysis: Interpretation of mass spectrum was conducted using the database of National Institute of Standards and Technology (NIST, USA). The database consists of more than 62,000 patterns of known compounds. The spectrum of the polystyrene constituents was matched with the spectrum of the known components stored in the NIST library. The chromatography results showed that some toxic polystyrene constituents) (O-xylene, Benzeethanol, tetradecanoic acid, cis-9-Hexadecenal) were removed).

Although plastic polymers have contributed to life in several ways, their persistence in the environment is doing more harm than good. For instance, due to indiscriminate dumping of solid waste, plastics continuously pile up in drainage systems, most especially in developing nations, which can subsequently result in flooding after a heavy rainfall (Aruna and Shanthi, 2015). Accumulated plastics in the environment can enter the food chain. For instance, researchers at the University of Bristol in the United Kingdom subjected Mytilus galloprovincialis to polyethylene and polystyrene microplastics, both virgin and previously contaminated with pyrene. The authors' observed a wide range of molecular, biochemical, and cellular alterations in the organism (Avio et al., 2015a). Furthermore, microplastics have been found in the stomachs of fishes from the Mediterranean and in the liver and gastrointestinal tract of sardines and anchovies (Compa et al., 2018). Microplastics have been proven to have an impact on the physiology and health of marine zooplankton, such as lowering feeding rates and decreasing reproductive success (Cole et al., 2015). Three isolates obtained from digestate are Bacillus sp., Paenibacillus sp. and Bacillus sp. Liaquat et al. (2015) similarly isolated Bacillus spp. from digestate while they were working on characterization of consortium bacterial from an anaerobic digester for biogas production using cow dung as inoculum. Isolates CDOS2 and CDOS3 from cow dung were presumptively identified as Pseudomonas sp. and Alcaligens sp. respectively. Gupta et al. (2108) previously isolated Alcaligenes sp. from cow dung while they worked on the bioactivity of Alcaligenes spp. isolated from cow dung against certain human pathogens. As revealed by the total viable count (TVC) (Figure 2), all the isolates showed appreciable growth on polystyrene. The reduction in weight of the polystyrene residue (Figure 1) showed that all five isolates have an appreciable ability to degrade polystyrene. Isolates OS1 (Bacillus sp.) and CDOS2 (Pseudomonas sp.) have the highest degrading ability on polystyrene. Isolate OS1 had a 0.3 $\mathrm{g}$ reduction, followed by CDOS2 with a $0.28 \mathrm{~g}$ reduction in weight. This is close to the results of Atiq et al. (2010); they isolated and characterized polystyrene degrading bacteria from soil. They affirmed that the soil bacteria (Paenibacillus sp., Bacillus sp. and Pseudomonas sp.) were able to colonize and metabolize expanded polystyrene as a sole source of carbon. An increase in total viable count (TVC) by week five (Figure 2) could probably mean that during this week the bacteria were utilizing the polymer as a carbon source, which led to their increase in growth. Also, there was a drastic decline in TVC by week 6 . This may be that the bacteria, due to their metabolic activities, released toxic substances into the culture medium. The $\mathrm{pH}$ of all of the culture media differed slightly. One of the methods used to determine degradation in this study is the measurement of the dry weight of residual polystyrene. The dry weight shows that there was a reduction in the weight of polystyrene across all five bacterial isolates. The initial $1 \mathrm{~g}$ of the polystyrene that was introduced to the mineral salt medium (MSM) inoculated with Paenibacillus sp. was reduced to 0.79 g $(21 \%$ reduction) by week seven. The highest reduction (degradation) was by Bacillus sp. (OS1) with $0.70 \mathrm{~g}$ remaining ( $30 \%$ degradation). This is consistent with a previous study in which small pieces of polystyrene $(0.5-1 \mathrm{~cm})$ were inoculated into liquid media (nutrient broth and Bushnell Hass broth). The media contained Bacillus subtilis, Pseudomonas aeruginosa, Staphylococcus aureus, and Aspergillus niger; the set up was incubated for a month. The 
highest weight loss in polystyrene was by B. subtilis with $20 \%$ and $58.8 \%$ in nutrient broth and Bushnell Hass broth respectively (Asmita et al., 2015). In their work on the formation of biofilm and partial degradation of polystyrene by Rhodococcus ruber, Roi and Alex (2008) added $1 \mathrm{~g}$ of polystyrene flake to the mineral salts' medium. They recorded a $0.005 \mathrm{~g}$ and $0.008 \mathrm{~g}$ reduction in weight in four and eight weeks, respectively. In this present study, a $0.3 \mathrm{~g}$ reduction was recorded by week seven. The statistical analysis for the reduction in weight of the polystyrene residue was performed using analysis of variance (one-way ANOVA), and the results showed that the difference between the reduction in weight of the polystyrene residue in the experiment compared to the control is statistically significant $\quad($ alpha $=0.05, \quad \mathrm{P}$-value $=$ 0.000878). The dry weight of the residual polystyrene substrate in the medium inoculated with Aspergillus niger strain R62 could not be determined because the mycelia of the fungus could not be easily washed off. Roi and Alex (2008) in their study were able to measure dry weight even though actinomycete mycelium was present because they used polystyrene flakes, which are more distinct than the polystyrene powder used in this present study. The chromatography analysis results show that the control chromatogram has twenty-seven peaks which most likely represented polystyrene constituents. The separation of the polystyrene may be because of the heating pre-treatment of the polystyrene substrate during autoclaving. The highest peak in the control and across all the cultures is represented by 9octadecenoic acid methyl ester (methyl oleate). It is an aliphatic unsaturated hydrocarbon used as a plasticizer, stabilizer, and colorant. It is an irritant, and combustible. It was utilized by Pseudomonas sp. (CDOS2). Its peak height in the control was 3151609 and was reduced to 1989399 . Other abundant compounds are dodecahydro-3, 8, and 11a-tetramethyl (5H-3,5a-Epoxynaphth [2,1-c] oxepin), cis-9hexadecenal, n-hexadecanoic acid, hexadecanoic acid methyl ester, and dodecanoic acid. Cis-9-hexadecenal is a toxic compound for aquatic life. But it was completely utilized by Pseudomonas sp. (CDOS2) and Bacillus sp. (2OS), but the fungus could not utilize it. O-xylene, diethyl phthalate, phenol 3-pentadecyl and benzene ethanol (phenyl ethanol) are among the toxic constituents of the polystyrene polymer that were utilized. Phenol 3-pentadecyl was completely removed by $2 \mathrm{OS}$ (Bacillus sp.), while the peak height of 1474637 in the control was reduced to 783028 and 904120 by Aspergillus niger strain R62 and CDOS2 (Pseudomonas sp.) respectively. O-xylene is one of the BTEX (Benzene, toluene, ethyl benzene, and xylene) is a volatile simple aromatic hydrocarbon present in crude oil and refined products such as gasoline. Any member of the BTEX is insoluble in water, and this makes them a major source of environmental pollution. The Pseudomonas sp. used in this present study was isolated from cow dung, although it was unable to oxidize the o-xylene. However, the chromatography results showed that there was ortho-cleavage which resulted in a change of the methyl group from carbon two $\left(\mathrm{C}_{2}\right)$ to carbon four $\left(\mathrm{C}_{4}\right)$. This is consistent with the report of El-Naas et al. (2014). They isolated Pseudomonas sp. from soil taken from the edge of a polluted creek in Austin, Texas, with p-xylene as the sole carbon source. This same bacterium was unable to oxidize o-xylene. ElNaas et al. further explained that the inability of the bacterium that utilized $\mathrm{p}$-and $\mathrm{m}$-xylene to degrade oxylene may be due to the position of the methyl group on the aromatic ring. In this study, the fungus Aspergillus niger was able to degrade o-xylene (1,2dimethylbenzene). Despite the low concentration of Diethyl phthalate, the microorganisms in this study could not utilize it appreciably. However, the reduction in peak heights may be an indication that Aspergillus niger and Bacillus sp. (2OS) attacked it. The peak height of the constituent in the control was $262474 \mathrm{~cm}$ and was reduced to $176300 \mathrm{~cm}$ and 205708 $\mathrm{cm}$ by the fungus and the bacterium, respectively. Singh et al., 2017, also reported that species of Pseudomonas isolated from plastic-contaminated soil utilized dimethyl phthalate (DEM) more than diethyl phthalate (DEP). The reduction in peak height as evident by the chromatography results in this study might probably be an indication that the organisms were able to extract some carbons from DEP. If the experimental period were extended, the organisms would likely oxidize DEP. Diethyl phthalate is a major toxic threat to plastic polymers. Continuous exposure to phthalate esters can lead to a serious health effect like male hormone disruption, which can lead to low sperm count and quality. They also cause low birth weight and reduce female fertility. It has been reported that phthalate esters can be released during use from the packaging materials and contaminate the content such as foods, water, and beverages (Sopheak et al., 2015). Similarly, Kanchanamayoon et al. (2012) also reported that the extracted phthalate esters from bottled drinking water are at a concentration higher than the maximum admissible concentration and that continuous exposure to these compounds may cause cancer. However, in another report by Fotopoulou and Karapanagiotis (2017), the expiry dates on bottle or sachet water are not for the water but the plastics. They used the expiry date to determine the age of the plastic. Benzeneethanol (2-phenyl ethanol), one of the polystyrene constituents, was reported by Niall et al., 2002 to be one of the transformation products in the aerobic degradation of styrene (polystyrene 
monomer). They also observed that the growth of Pseudomonas sp. on the styrene monomer led to the formation of 2-Phenyl ethanol from styrene oxide. Although the authors did not report that Pseudomonas sp. degrades 2-phenyl ethanol, they did observe that a specie of Xanthobacter was able to grow on 2-phenyl ethanol and styrene oxide as sole carbon sources. Contrastingly, in this study, both Pseudomonas sp. and Bacillus sp. were able to utilize 2-phenyl ethanol. But the fungus, Aspergillus niger strain R62 (MK54 2010) was unable to metabolize it. The use of the fungus (Aspergillus niger) isolated from abattoir effluent and bacterial isolates from cow dung (Pseudomonas sp and Alcaligens sp) and digestate (Bacillus sp., Paenibacillus sp., and Bacillus sp.) gave positive results towards degrading polystyrene polymer. The disappearance of the polystyrene constituents and the reduction in peak heights as shown by gas chromatography analysis is an indication that the bacterial isolates and the fungus were able to extract some carbon from the complex molecule of polystyrene. The highest percentage of degradation (30\%) was recorded after periods of seven weeks of the experimental set up. The slow or nonbiodegradability of synthetic polymers may be because of their hydrophobicity, degree of crystallinity, surface topography and large molecular size (Webb et al., 2013).

Polymers with carbon-carbon bonds in their backbone such as polystyrene, polypropylene, polyolefins, polyvinyl chloride (PVC) and polyethylene are more difficult to biodegrade compared to polymers with heteroatomic backbones like polyurethane and polyethylene terephthalates. The material properties and, as a result, the applications of polymers with a carbon-carbon bond backbone are greatly influenced (Anne et al., 2019).

To the best of our knowledge, the bacteria and fungus used in this study showed the ability to colonize and appreciably utilize the polystyrene polymer compared to previous reports. This, in our own opinion, is credited to these tropical microorganisms which are well adapted to harsh environmental conditions (Buraimoh et al., 2015) such as the colon of a cow, anaerobic digesters, and abattoir effluents where the microbial isolates used in this study were obtained. In addition, the reduction of polystyrene to powder allowed for increased surface area for easy attack by the microbial isolates.

Conclusion: The microorganisms utilized in this study have shown an appreciable ability to utilize polystyrene, a major plastic found in various environments. Cis-9-hexadecenal is a toxic compound for aquatic life. But it was completely utilized by
Pseudomonas sp. (CDOS2) and Bacillus sp. (2OS). Oxylene, diethyl phthalate, phenol 3-pentadecyl and benzene ethanol (phenyl ethanol) are among the toxic constituents of the polystyrene polymer that were utilized by these tropical microorganisms. If properly harnessed, they could contribute to environmental friendly solutions that will address major challenges associated with persistent plastics and their attendant impacts on health, land, and aquatic ecosystems.

\section{REFERENCES}

Anne, S; Jonas, H; Philipp, G. (2019). Group 13Group 15 Element Bonds Replacing CarbonCarbon Bonds in Main Group Polyolefin Analogs. In: Evamarie Hey-Hawkins,Muriel Hissler, Editors: Smart inorganic polymers, pp. 13-15

Aruna, M; Shanthi, V (2015). Biodegradation of Plastics - A Brief Review. Int J Pharm Sci Rev Res, 31(2):204-209

Atiq, N; Ahmed, S; Ishtiaq, M; Andleeb, S; Ahmed, B; Geoffrey, R (2010). Isolation and identification of Polystyrene biodegrading bacteria from soil. AJMR, 4(14):1538-1541

Asmita, A; Shubhamsingh, T; Tejashree, S (2015). Isolation of Plastic Degrading Micro-organisms from Soil Samples Collected at Various Locations in Mumbai, India Int Res J Environ Sci., 4:77-85

Avio, CG; Gorbi, S; Milan, M; Benedetti, M; Fattorini, D; d'Errico, G; Pauletto, M; Bargelloni, L; Regoli, F. (2015a). Pollutant's bioavailability and toxicological risk from microplastics to marine mussels. Environ. Pollut. 198:211-222.

Bratovcic, A (2019). Degradation of Micro- and NanoPlastics by Photocatalytic Methods. J. Nanosci. Nanotechnol., 3:304-313

Buraimoh, OM; Ilori, MO; Amund, OO; Frederick, MC; Grewal, SK (2015). Assessment of bacterial degradation of lignocellulosic residues (sawdust) in a tropical estuarine microcosm using improvised floating raft equipment. Int. Biodeterior. Biodegrad., 104:186-193

Chae, Y; An, YJ (2017). Effects of micro and nanoplastics on aquatic ecosystems: current research trends and perspectives. Mar. Pollut. Bull., 124:624-632

Cole, M; Lindeque, P; Fileman, E; Halsband, C; Goodhead, R; Moger, J; Galloway, TS. (2013). 
Microplastic ingestion by zooplankton. Environ. Sci. Technol. 47:6646-6655.

Compa, C; Ventero, A; Iglesias, M; Deudero, S (2018). Ingestion of microplastics and natural fibres in Sardina pilchardus (Walbaum, 1792) and Engraulis encrasicolus (Linnaeus, 1758) along the Spanish Mediterranean coast. Mar. Pollut. Bull., 128:89-96

El-Naas, MH; Acio, J. A; El Telib, AE. (2014). Aerobic biodegradation of BTEX: Progresses and Prospects. J. Environ. Chem. Eng., 2(2):11041122.

Foley, CJ; Feiner, ZS; Malinich, TD; Höök, TO (2018). A meta-analysis of the effects of exposure to microplastics on fish and aquatic invertebrates. Sci. Total Environ. 631: 550-9

Fotopoulou, KN; Karapanagiotic (2017). Degradation of various plastics in the Environment. Environ. Chem., 10:11

Garcia, JM; Robertson, ML (2017). The future of plastics recycling. Science, 358:870-872

Gupta, KK; Rana, D. (2018). Bioactivity of Alcaligenes spp. isolated from cow dung against certain human pathogens. Environ. Conserv., 19(1\&2): 59-64

Horodytska, O; Valdés, FJ; Fullana, A. (2018). Plastic flexible films waste management - A state of art revie. Waste Manag, 77:413-425.

http:// www.natgeo.org/plastics. Date accessed: 1107-2019

Jia, X; Qin, C; Friedberger, T; Guan, Z; Huang, Z (2016). Efficient and selective degradation of polyethylenes into liquid fuels and waxes under mild conditions. Sci. Adv, 2:501-591

Jonas, AAR; Willy VNP. (2019). Fishing marine debris in a northeast Brazilian beach: composition, abundance, and tidal changes. Mar. Pollut. Bul., 142:428-432.

Kanchanamayoon, W; Prapatpong, P; Chumwangwapee, S; Chaithongrat, S (2012). Analysis of phthalate esters contamination in drinking water samples. Afr. J. Biotechnol, 11(96):16263-16269
Lettieri, P; Al-Salem, S (2011). Thermochemical treatment of plastic solid waste. In: Letcher T. M, ValleroD, editors.Waste :A handbook for management; pp. 233-42

Liquant, R; Jamal, A; Tauseef, I; Quaresh, Z; Farooq, U; Imran, M; Ali, MI (2017). Characterization of Bacterial consortia from an anaerobic digester treating organic waste for biogas production. Pol. J. Environ. Stud., 26:709-716

Niall, DO; Kevin E. O; Alan, DWD. (2002). Biochemistry, genetics, and physiology of microbial styrene degradation. FEMS Microbiology Reviews, 26(4):403-417

Ragaert, K; Delva, L; Van Geem, K (2017). Mechanical and chemical recycling of solid plastic waste. J. Waste Manage. 69:24-58

Roi, S; Alex, S (2008). Biofilm formation and partial biodegradation of polystyrene by the actinomycete Rhodococcus ruber. Biodegradation, 19(6):851858

Singh, N; Dalal, V; Mahto, JK; Kumar, P (2017). Biodegradation of phthalic acid esters (PAEs) and in silico structural characterization of mono-2ethylhexyl phthalate (MHEP) hydrolase based on close structural homolog. J. hazard. Mater. 338:11-22

Sopheak, N; Anne, D; Richard, S; Andrea, P; Baghdad, O. (2015). Reliable quantification of phthalates in environmental matrices (air, water, sludge, sediment, and soil): a review.

Sci. Total Environ. 515:162-180

Urbanek, AK; Rymowicz, W; Mirończuk, AM (2018). Degradation of plastics and plastic-degrading bacteria in cold marine habitats. Appl. Microbiol. Biotechnol., 102: 7669-7678

van Cauwenberghe, L; Janssen, CR (2014). Microplastics in bivalves cultured for human consumption. Environ. Pollute. 193:65-70

Webb, HK; Jaimys, A; Crawford, RJ; Elena PI (2013). Plastic Degradation and Its Environmental Implications with Special Reference to Poly (ethylene terephthalate). Polymers, 5(1):1-18 